Original Paper http://ajol.info/index.php/ijbcs http://indexmedicus.afro.who.int.

\title{
Distribution spatiale des éléments traces métalliques dans les sols de la zone aurifère de Komabangou au Niger
}

\author{
Ousseini ZAKARIA IBRAHIM ${ }^{1}$, Abdourahamane TANKARI DAN-BADJO ${ }^{1 *}$, \\ Yadji GUERO $^{1}$, Farida MAISSORO MALAM IDI ${ }^{2}$, Cyril FEIDT $^{3}$, \\ Thibault STERCKEMAN ${ }^{4}$ et Guillaume ECHEVARRIA ${ }^{4}$
}

\author{
${ }^{1}$ Département Science des sols, Faculté d'Agronomie, Université Abdou Moumouni de Niamey, Niger. \\ ${ }^{2}$ Ecole Des Mines de l'Industrie et de la Géologie (EMIG) de Niamey, Niger. \\ ${ }^{3} U R$ AFPA, Université de Lorraine, INRA, France. \\ ${ }^{4}$ Laboratoire Sols et Environnement, Université de Lorraine, INRA, France. \\ *Auteur correspondant, E-mail: tankari@yahoo.fr
}

\section{REMERCIEMENTS}

Cette étude a été financée par le projet SIDA-UNESCO sur les mines abandonnées dans les pays d'Afrique subsaharienne (numéros de contrats : 4500279938 et 4500309527). Les auteurs remercient SIDAUNESCO pour ce soutien financier.

\section{RESUME}

Cette étude s'inscrit dans un projet de recherche portant sur l'évaluation des impacts environnementaux et sanitaires de l'orpaillage dans la zone de Komabangou au Niger. L'objectif est de déterminer les niveaux de contamination des éléments traces dans les sols et d'analyser leur distribution spatiale. Des échantillons des sols ont été prélevés en 2016 dans 27 localités dont 24 sur différents sites de la zone aurifère de Komabangou et 3 sur sites témoins. Les concentrations en éléments traces ( $\mathrm{As}, \mathrm{Cd}, \mathrm{Co}, \mathrm{Cr}, \mathrm{Cu}, \mathrm{Ni}, \mathrm{Pb}$ et $\mathrm{Zn}$ ) dans les sols ont été déterminées par spectrométrie de masse avec plasma à couplage inductif (ICP-MS). La pollution des sols a été évaluée en calculant les index de pollution (IP) des sols. Les résultats montrent que les concentrations en éléments traces métalliques (ETM) dans les sols diffèrent en fonction du site et du métal considéré. Les cartes de répartition spatiale montrent une importante concentration des éléments traces tels que $\mathrm{As}, \mathrm{Cd}, \mathrm{Pb}$ et $\mathrm{Zn}$ au centre de la zone aurifère. Plusieurs de ces teneurs en ETM sont supérieures aux seuils réglementaires. Aussi sur les 27 sites étudiés, 12 ont des valeurs d'IP supérieures à 1 révélant ainsi une contamination des sols par plusieurs éléments. Ces résultats montrent une accumulation des métaux dans les sols qui peuvent persister dans l'environnement. Cela peut poser de sérieux risques sanitaires pour l'Homme. Pour prévenir tout risque, un plan de surveillance de la contamination des sols devrait être mis en place et il faudrait envisager de traiter les sites pollués.

(c) 2019 International Formulae Group. All rights reserved.

Mots clés : Orpaillage, sols, contamination, éléments traces métalliques, valeurs limites, Komabangou, Niger. 


\title{
Spatial distribution of metallic trace elements in soils of Komabangou gold zone in Niger
}

\begin{abstract}
This study is part of research project on environmental and health impacts of gold mining in Komabangou area of Niger. The objective is to determine pollution levels of trace elements in soils and to analyze their spatial distribution. Soil samples were collected in 2016 at 27 localities, including 24 at different sites in Komabangou gold zone and 3 at control sites. Concentrations of trace elements (As, $\mathrm{Cd}, \mathrm{Co}, \mathrm{Cr}, \mathrm{Cu}, \mathrm{Ni}$, $\mathrm{Pb}$ and $\mathrm{Zn}$ ) in soils were determined by Inductively Coupled Plasma Mass Spectrometry (ICP-MS). Soil pollution was assessed by calculating soil pollution index (PI). The results show that metal trace element concentrations in soils differ depending to the site and the metal. Spatial distribution maps show a high concentration of trace elements such as $\mathrm{As}, \mathrm{Cd}, \mathrm{Pb}$ and $\mathrm{Zn}$ in the center of the gold zone. Many concentrations of trace elements in soils are above regulatory thresholds. Also, of the 27 sites studied, 12 have PI values greater than 1, revealing soil contamination by several elements. These results show an accumulation of metals in soils, which can persist in the environment. This can pose serious health risks for humans. To prevent any risk, soil contamination monitoring plan should be put in place and consideration should be given to treating polluted sites.
\end{abstract}

(C) 2019 International Formulae Group. All rights reserved.

Keywords: Gold panning, soils, contamination, trace elements, limit values, Komabangou, Niger.

\section{INTRODUCTION}

L'exploitation minière est devenue une activité économique majeure dans plusieurs pays africains et constitue une source de revenus pour un nombre important des populations tout en suscitant de nombreux espoirs de développement. La promulgation de nouvelles législations minières vers la fin des années 80, dans la plupart des pays, a favorisé l'investissement privé dans ce secteur (Lamine, 2011). Le Niger comme beaucoup de ces pays, dispose d'énormes potentialités minières pouvant constituer une importante base pour son économie, particulièrement : le pétrole dans sa partie orientale (AGADEM), l'uranium dans sa partie septentrionale (AIR) et l'or dans sa partie occidentale (LIPTAKO), etc. Cependant, l'exploitation de ces ressources reste, le plus souvent, sans retombées économiques sensibles pour la population locale et constitue de plus une source de dégradation de l'environnement, mettant en danger certains patrimoines vitaux (sols, eaux, végétation...etc.). C'est ainsi que, derrière l'exploitation de certaines ressources, de nombreux sites restent potentiellement contaminés par diverses substances chimiques généralement nocives, notamment les éléments traces métalliques (ETM). La contamination du sol peut résulter de l'accumulation de métaux lourds et métalloïdes comme le plomb, le cadmium, le mercure et le nickel résultant de l'industrialisation, des dépôts atmosphériques, des déchets, des déversements pétrochimiques, des résidus miniers, des résidus de combustion du charbon, de l'essence et des engrais. Les métaux lourds rejetés dans l'environnement par les activités humaines ne subissent pas de dégradation microbienne ou chimique, et peuvent donc persister dans les sols longtemps après leur libération (Aucejo et al., 1997). L'introduction de ces contaminants dans les sols peut entraîner la détérioration et la disparition de certaines fonctions du sol, avec une possible contamination des ressources (Bougherara et al., 2011). Des concentrations élevées de métaux lourds dans le sol constituent une menace possible pour la santé humaine puisque les humains peuvent être exposés par ingestion accidentelle, par contact direct et par inhalation de poussières en suspension. 
Des éléments traces comme l'arsenic et le plomb ont des effets cancérigènes (Jaishankar et al., 2014). Les autres métaux comme le cuivre et le zinc sont des nutriments essentiels pour la santé humaine mais peuvent être toxiques à fortes concentrations (Rahman et al., 2014).

L'exposition au plomb entraîne des problèmes de santé : avortement chez les femmes, retards de développement et dommages au système nerveux chez les enfants. Le plomb est un métal particulièrement dangereux qui n'a aucun rôle biologique et affecte négativement les enfants de façon significative. L'exposition postnatale et prénatale au plomb est associée au neurodéveloppement de l'enfant (Agusa et al., 2006). Les études sur l'exposition postnatale montrent des effets à long terme sur le quotient intellectuel de l'enfant, ainsi que sur l'attention et l'inhibition (Lanphear et al., 2005). L'exposition environnementale au cadmium est associée à un risque accru de cancer et de mortalité cardiovasculaire chez les hommes, mais pas chez les femmes (Lanphear et al., 2005). L'exposition chronique au cadmium peut augmenter la mortalité et raccourcir l'espérance de vie (Freiberg, Elinder, Kjellstrom \& Nordberg, 1986).

Les problèmes posés par la dispersion des métaux lourds dans l'environnement suscitent l'intérêt de la communauté scientifique depuis maintenant de nombreuses années (Breuer et al., 2004; Smouni et al., 2010; Tankari Dan-Badjo et al., 2014; Lu et al., 2015; Toure et al., 2016; Adeyi et Babalola, 2017; Oymak et al., 2017; Chouti et al., 2018).

Le secteur minier notamment l'exploitation aurifère constitue une des importantes sources de dispersion de ces contaminants dans les sols. Au Niger, l'exploitation artisanale aurifère ou orpaillage est devenue une activité en pleine expansion. Dans la région de Tillabéri, par exemple, et à Komabangou en particulier, l'or est exploité de manière traditionnelle durant des décennies avec utilisation des substances dangereuses comme le mercure et le cyanure. Les travaux d'exploitation (creusement) et de prétraitement (concassage, broyage) libèrent une quantité notoire des éléments traces métalliques dans l'environnement. Ainsi, durant cette exploitation aurifère, des grandes quantités des déchets solides sont générées et entreposées sous forme de terrils. Ces déchets miniers abandonnés, sans aménagement et soumis aux aléas climatiques notamment les vents et les précipitations, représentent une source de contamination de l'environnement (eau, sols, végétaux) à proximité de ces sites par les éléments traces métalliques (ETM) et le cyanure.

Nos travaux de recherche ont révélé une pollution métallique des eaux prélevées dans la zone de Komabangou (Tankari DanBadjo et al., 2015). La présente étude s'inscrit dans ce sens et vise à déterminer les teneurs en ETM dans les sols de Komabangou et ses alentours et d'analyser leur distribution spatiale.

\section{MATERIEL ET METHODES}

\section{Description du site d'étude}

Le site d'orpaillage de Komabangou (Figure 1) est situé dans la région de Tillabéri, à environ $150 \mathrm{~km}$ au Nord-Ouest de la ville de Niamey. De Niamey, il est accessible via la route bitumée de Tillabéri, ou celle de Namaro, le bac Farié, la route nationale $\mathrm{N}^{\circ} 103$ vers Dargol et Boura, puis par la piste (26 km) latéritique à sablonneuse jusqu'au site. C'est une vaste zone à forte densité d'indices et d'anomalies géochimiques aurifères. Se trouvant entre la latitude $14^{\circ} 01^{\prime} 41^{\prime}$ 'et $14^{\circ} 07^{\prime} 56^{\prime \prime}$ Nord et la longitude $01^{\circ} 02^{\prime} 12^{\prime \prime}$ 'et $01^{\circ} 10^{\prime} 00^{\prime}$ ' Est, elle couvre une superficie de $157 \mathrm{~km}^{2}$ avec une population estimée à 36937 habitants répartis dans 18 villages du terroir (INS, 2010). C'est un site extrêmement riche en veines de quartz de plusieurs générations successives, dont certaines très riches en or, qui est exploité de manière traditionnelle. Les sites d'exploitation se présentent par une émergence sauvage et très désordonnée des puits et des carrières comme des galeries des bousiers. Sur ces sites, on observe des terrils de roches ou sables stériles rejetés lors de creusage des puits à la recherche de la roche 
utile contenant de l'or (Tankari Dan-Badjo et al., 2014).

\section{Prélèvement des échantillons}

Le compartiment étudié est le sol de komabangou. Il a été divisé en trois catégories à savoir: sol zone aurifère, sol zone périphérique et le sol témoin. L'intérêt scientifique porte sur les éléments traces métalliques que sont l'arsenic (As), le cadmium $(\mathrm{Cd})$, le cobalt $(\mathrm{Co})$, le chrome $(\mathrm{Cr})$, le cuivre $(\mathrm{Cu})$, le nickel $(\mathrm{Ni})$, le plomb $(\mathrm{Pb})$, le zinc ( $\mathrm{Zn})$. Pour le besoin de collecte de données, des campagnes de prélèvement des échantillons de sol ont été faites au niveau de chacune de ces catégories précitées. Les échantillons des sols ont été prélevés au niveau des 27 sites dans le but de déterminer leurs teneurs en éléments traces métalliques précédemment cités. Ces prélèvements ont été effectués à l'aide d'une tarière sur une profondeur comprise entre 0 et $30 \mathrm{~cm}$. Au niveau de chaque site, un échantillon composite $(0,5$ à $1 \mathrm{~kg}$ de sol) est réalisé à partir de 5 prélèvements élémentaires. Cet échantillon est conditionné en sachet plastique propre et bien identifié. Au total 27 échantillons composites de sol ont été constitués dont 9 dans la zone aurifère, 15 en zone périphérique et 3 hors zone aurifère (témoin). La Figure 2 donne la répartition spatiale des échantillons des sols prélevés.

\section{Extraction et dosage des métaux dans les sols}

Les échantillons de sols ont été séchés à une température ambiante $\left(<40^{\circ} \mathrm{C}\right)$, broyés et tamisés à $2 \mathrm{~mm}$ au laboratoire du Département Science du Sol de la Faculté d'Agronomie de Niamey. L'extraction et le dosage des ETM totaux dans les sols ont été réalisés au Centre de Recherches Pétrochimiques et Géochimiques de Nancy (France) par le Service d'Analyse des Roches et des Minéraux (SARM). La mise en solution de $\mathrm{As}, \mathrm{Cd}, \mathrm{Co} \mathrm{Cr}, \mathrm{Cu}, \mathrm{Ni}, \mathrm{Pb}$ et $\mathrm{Zn}$ a été effectuée à l'aide d'un mélange d'acide fluorhydrique et perchlorique $\left(\mathrm{HClO}_{4}\right)$ selon la norme NF $X$ 31-151. L'analyse est effectuée par spectrométrie de masse avec plasma à couplage inductif.

\section{Calcul de l'index de pollution par les ETM dans les sols}

La contamination par les métaux lourds à la surface des sols, en particulier dans les sites miniers, est associée à un cocktail de contaminants plutôt qu'à un seul métal (Lee et al., 2001). Ainsi, le concept d'un index de pollution (IP) des sols a été introduit dans de nombreuses études pour identifier la contamination multiéléments qui se traduit par une augmentation de la toxicité métallique (Chon et al., 1998; Smouni et al., 2010; Tankari Dan-Badjo et al., 2013). Il s'agit d'un critère permettant d'évaluer la toxicité globale d'un sol contaminé.

Selon Chon et al. (1998), l'index de pollution du sol est calculé à partir de la moyenne des rapports des concentrations en métaux dans les échantillons de sol par rapport aux valeurs directives limites. Ces valeurs limites correspondent aux niveaux supposés tolérables dans le sol. Ainsi, l'index de pollution est calculé par l'équation suivante :

IP

$[(\mathrm{As} / 6+\mathrm{Cd} / 1+\mathrm{Co} / 30+\mathrm{Cr} / 150+\mathrm{Cu} / 100+\mathrm{Ni} / 50+$ $\mathrm{Pb} / 100+\mathrm{Zn} / 300) / 8]$

IP > 1 correspond à un sol pollué par plusieurs métaux.

\section{Analyses statistiques des résultats}

Le logiciel Microsoft Excel a été utilisé pour l'analyse descriptive des résultats et la corrélation entre les variables. C'est ainsi que la moyenne, l'écart-type, la valeur maximale et la valeur minimale ont été déterminés pour chaque élément chimique analysé. Le logiciel $\mathrm{R}$ a été utilisé pour faire les graphiques. Les logiciels de cartographie Arc-Gis et Arcview ont été utilisés pour la production des cartes de répartition spatiale des teneurs en ETM ayant dépassé les valeurs réglementaires admises, dans le sol de Komabangou. 


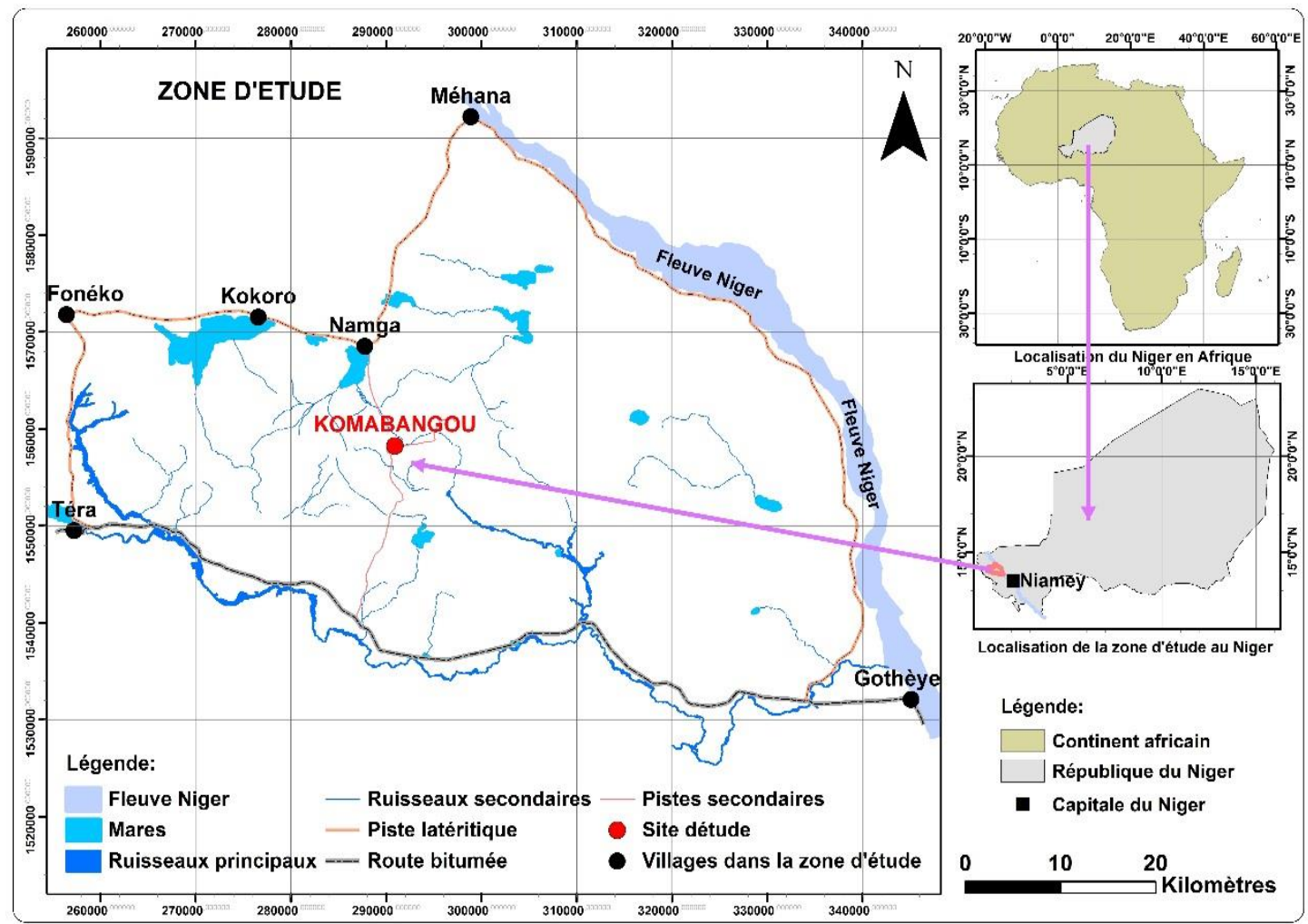

Figure 1 : Localisation du site aurifère de Komabangou.

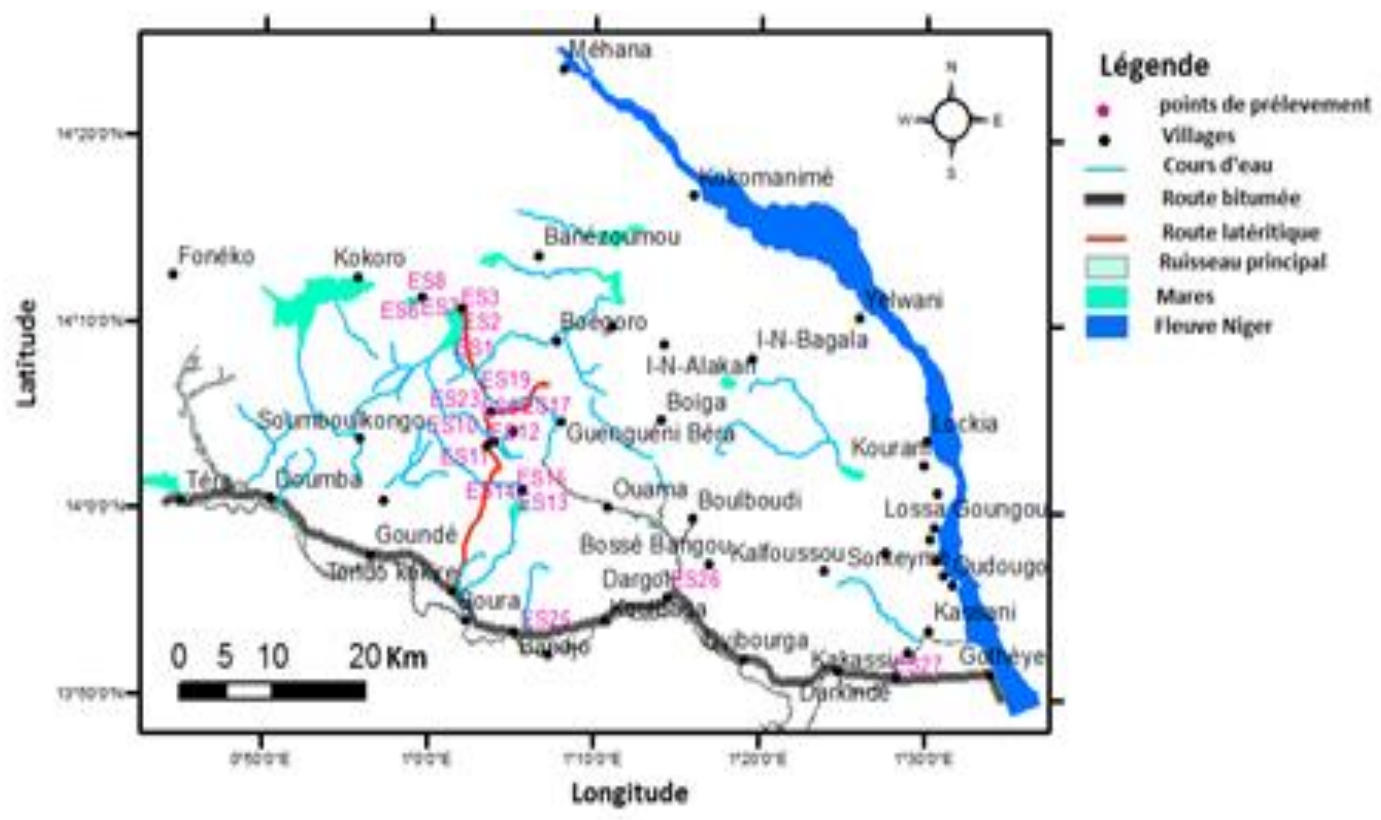

Figure 2 : Répartition spatiale des points de prélèvement des sols dans la zone aurifère de Komabangou et ses environs. 


\section{RESULTATS}

\section{Concentrations en ETM dans les échantillons du sol}

Les concentrations de chaque métal dans les échantillons de sol, tous sites confondus, sont représentées sur la Figure 3 qui traduit une variabilité inter-sites des teneurs en fonction du métal considéré. En effet, l'examen de cette Figure, nous permet de faire 3 types de constat :

- Pour certains métaux ( $\mathrm{Cd}, \mathrm{Co}$ et $\mathrm{Ni})$, il $\mathrm{y}$ a très peu de variabilité de concentrations, ce qui traduit un niveau de pollution similaire entre les différents sites étudiés.

- A l'inverse, on constate une forte variabilité des concentrations en As, $\mathrm{Pb}$ et $\mathrm{Zn}$. Pour ces trois éléments, les valeurs médianes sont de $4,67 \mathrm{mg} / \mathrm{kg}$, $7,20 \mathrm{mg} / \mathrm{kg}$ et $51,53 \mathrm{mg} / \mathrm{kg}$ respectivement.

- Pour le $\mathrm{Cr}$ et le $\mathrm{Cu}$, on constate une plage de variabilité intermédiaire.

Les valeurs de concentration en éléments traces dans les sols sont très disparates et varient de 0,541 à $555,585 \mathrm{mg} / \mathrm{kg}$ pour l'As ; 0,037 à $1,434 \mathrm{mg} / \mathrm{kg}$ pour le $\mathrm{Cd}$; 1,240 à 27,146 pour le $\mathrm{Co} ; 18,699$ à 257,798 $\mathrm{mg} / \mathrm{kg}$ pour le $\mathrm{Cr} ; 3,859$ à 175,535 pour le $\mathrm{Cu}$ ; 5,402 à $126,984 \mathrm{mg} / \mathrm{kg}$ pour le $\mathrm{Ni}$; et 2,711 à $6576,699 \mathrm{mg} / \mathrm{kg}$ et 7,558 à $468,960 \mathrm{mg} / \mathrm{kg}$ respectivement pour le $\mathrm{Pb}$ et le $\mathrm{Zn}$. La concentration moyenne la plus élevée est obtenue pour le $\mathrm{Pb}$, suivie par ordre décroissant de celles du $\mathrm{Zn}$, As, $\mathrm{Cr}, \mathrm{Cu}, \mathrm{Ni}$, $\mathrm{Co}$ et $\mathrm{Cd}$.

La Figure 4 montre les teneurs moyennes $(\mathrm{mg} / \mathrm{kg})$ en éléments traces dans les échantillons des sols selon qu'ils soient prélevés en zone minière, en périphérie de mine ou en hors zone minière. L'analyse de cette figure révèle clairement que les sols miniers de Komabangou sont plus riches en $\mathrm{As}, \mathrm{Cu}, \mathrm{Pb}$ et $\mathrm{Zn}$ comparés aux sols en périphérie de mine et sols hors zone minière. Les sols en périphérie de mine présentent des teneurs en éléments traces plus élevées que celles des sols hors zone minière suggérant ainsi que l'exploitation artisanale de l'or dans la zone de Komabangou pollue les sols en éléments traces.

\section{Teneurs en arsenic dans les échantillons des sols}

La Figure 5 montre la répartition spatiale des teneurs en ETM des sols étudiés. Les teneurs en As dans les échantillons des sols analysés varient d'une valeur inférieure à la limite de détection à $555,58 \mathrm{mg} / \mathrm{kg}$. La teneur en As la plus élevée est obtenue au niveau du site «Grillage Bidon» (ES17). Douze (12) sites sur 27 présentent des teneurs supérieures à la moyenne mondiale des sols non contaminés en As $(6 \mathrm{mg} / \mathrm{kg})$. Les valeurs guides pour les sols contaminés en As sont fixées à $20 \mathrm{mg} / \mathrm{kg}$ et $29 \mathrm{mg} / \mathrm{kg}$ respectivement par la législation italienne et par l'agence hollandaise pour la protection de l'environnement. Neuf (9) des échantillons analysés présentent des teneurs au-dessus de ces normes. Ces échantillons sont ES16, ES17, ES18, ES19, ES20, ES21 ES22, ES23, ES24, correspondant successivement aux sites d'orpaillage de Grillage El Saadou, Grillage Bidon, Grillage Morey, Grillage Baba Djallo, Nakia Sossoriya, Maijamaa Sossoriya, Abdallah Sossoriya, Banbara Sosssriya et Gawado Sossoriya ; tous situés à Komabangou. Les teneurs en As des échantillons prélevés hors zone minière (ES25, ES26 et ES27) sont largement inférieures à celles des sites de komobangou.

\section{Teneurs en cadmium dans les échantillons des sols}

Les teneurs en Cd dans les sols étudiés varient de 0,037 à $1,434 \mathrm{mg} / \mathrm{kg}$. La Figure 6 donne la répartition spatiale des teneurs en $\mathrm{Cd}$ des échantillons étudiés. La valeur la plus élevée est obtenue dans l'échantillon (ES23) du site Bambara Sosssriya, et est la seule valeur supérieure au seuil fixé par l'agence britannique de l'environnement comme valeur de référence pour les sols de zones urbaines. Elle est également supérieure à celles obtenues en Chine par Huang et al. (2013) sur les sols miniers d'exploitation de l'or et du fer $\mathrm{du}$ réservoir de Miyun et de Beijing qui sont respectivement de $0,85 \mathrm{mg} / \mathrm{kg}$ et $0,54 \mathrm{mg} / \mathrm{kg}$. 


\section{Teneurs en cobalt dans les échantillons des sols}

Les concentrations en Co obtenues dans les sols étudiés varient de 1,240 à 27,146 $\mathrm{mg} / \mathrm{kg}$. Sur l'ensemble des échantillons analysés, il n'y a aucune concentration en Co supérieure à la valeur règlementaire (30 $\mathrm{mg} / \mathrm{kg}$ ) définie par l'Association Française de Normalisation sous la norme NFU 44-041. Seuls certains échantillons prélevés à Namga (ES1, ES2, ES5) ont des teneurs en Cobalt sensiblement égales à cette valeur limite. La Figure 7 présente la répartition spatiale des concentrations en cet élément dans les sols.

\section{Teneurs en chrome dans les échantillons des sols}

La Figure 8 présente la répartition spatiale des teneurs en $\mathrm{Cr}$ dans les échantillons prélevés. Ces teneurs vont de 18,699 à $257,79 \mathrm{mg} / \mathrm{kg}$. Les teneurs en $\mathrm{Cr}$ les plus élevées sont détectées au niveau du village de Namga dans les échantillons (ES1, ES2, ES5) prélevés et sont supérieures au seuil de référence $(150 \mathrm{mg} / \mathrm{kg})$ marquant la différence entre sols non contaminés et sols contaminés en $\mathrm{Cr}$ selon la norme française NF U44-041.Seul l'échantillon ES11 prélevé à Komabangou Boura a une teneur $(143,277$ $\mathrm{mg} / \mathrm{kg}$ ) tendant vers cette valeur limite.

\section{Teneurs en cuivre dans les échantillons des sols}

Les teneurs en $\mathrm{Cu}$ obtenues varient de $3,859 \mathrm{mg} / \mathrm{kg}$ pour l'échantillon ES25 prélevé hors zone minière à $175,535 \mathrm{mg} / \mathrm{kg}$, teneur de l'échantillon ES22 du site Abdallah Sossoriya. Sur les 27 échantillons analysés, 13 présentent des teneurs en $\mathrm{Cu}$ supérieures à la moyenne mondiale des sols non contaminés en $\mathrm{Cu}$ (30 $\mathrm{mg} / \mathrm{kg}$ ) et seulement 2 (ES2 et ES22) prélevés respectivement à Namga et Abdallah Sossoriya dépassent le niveau tolérable de concentration de $\mathrm{Cu}$ dans les sols (100 $\mathrm{mg} / \mathrm{kg}$ ). La Figure 9 donne la répartition spatiale des teneurs en $\mathrm{Cu}$.

\section{Teneurs en Nickel dans les échantillons des sols}

Les teneurs en Ni dans les sols étudiés varient de 5,402 (zone non minière de Bandjo) à 126,984 mg/kg (Namga). Ces valeurs sont supérieures à $5 \mathrm{mg} / \mathrm{kg}$, qui est une valeur limite séparant un sol contaminé en $\mathrm{Ni}$ et un sol non contaminé selon les valeurs guides relatives aux teneurs moyennes mondiales des métaux dans les sols non contaminés. En comparant les teneurs en $\mathrm{Ni}$ avec la norme française NF U44-041 (Baize et Paquereau, 1997) fixée à $50 \mathrm{mg} / \mathrm{kg}$, seul le village de Namga serait pollué. La Figure 10 donne la répartition spatiale des teneurs en $\mathrm{Ni}$.

\section{Teneurs en Plomb dans les échantillons des sols}

La Figure 11 donne la répartition spatiale des teneurs en $\mathrm{Pb}$. Les concentrations de $\mathrm{Pb}$ dans nos échantillons de sols varient 2,711 à $6576,699 \mathrm{mg} / \mathrm{kg}$. La teneur la plus faible en $\mathrm{Pb}$ est obtenue dans une zone non minière (Bandjo) et la plus forte est enregistrée dans la zone minière Banbara sossoriya (village de Komabangou).19 échantillons sur 27 présentent des concentrations en $\mathrm{Pb}$ inférieures à la moyenne mondiale des teneurs en $\mathrm{Pb}$ des sols non contaminés, établie à $35 \mathrm{mg} / \mathrm{kg}$. Les teneurs supérieures à la valeur limite de $100 \mathrm{mg} / \mathrm{kg}$ fixée par l'OMS dans les sols sont toutes enregistrées sur des sites miniers de Komabangou.

\section{Teneurs en Zinc dans les échantillons des sols}

La Figure 12 présente la répartition spatiale des teneurs en $\mathrm{Zn}$ dans les sols étudiés. Les teneurs en zinc varient de 7,558 à $468,960 \mathrm{mg} / \mathrm{kg}$. La valeur la plus élevée est obtenue sur le site de cyanuration Grillage El Saadou dépassant largement la moyenne mondiale des teneurs en $\mathrm{Zn}$ dans les sols non contaminés $(90 \mathrm{mg} / \mathrm{kg})$. Comparées à la norme réglementaire hollandaise de 140 $\mathrm{mg} / \mathrm{kg}$ pour les sols contaminés, 4 échantillons (ES16, ES17, ES19, ES21 et ES23) tous les échantillons prélevés dans la zone minière de Komabangou présentent des teneurs en $\mathrm{Zn}$ dans les sols supérieures à cette norme. Les échantillons prélevés dans les zones non minières ont tous des teneurs en $\mathrm{Zn}$ inférieures à la norme. 


\section{Index de pollution par les ETM dans les} sols

Les index de pollution (IP) des sols ont été calculés pour les différents sites de prélèvement, les résultats sont donnés par le Tableau 1.

L'analyse de ce tableau nous révèle des valeurs d'IP variant de 0,06 à 17,84 en fonction du site considéré. L'index le plus bas est obtenu au niveau de la zone non minière de Bandjo indiquant une faible accumulation des métaux lourds sur ce site. Sur les 27 sites étudiés, 12 ont des valeurs d'IP supérieures à 1 , révélant ainsi la contamination multiple de leurs sols par les ETM.

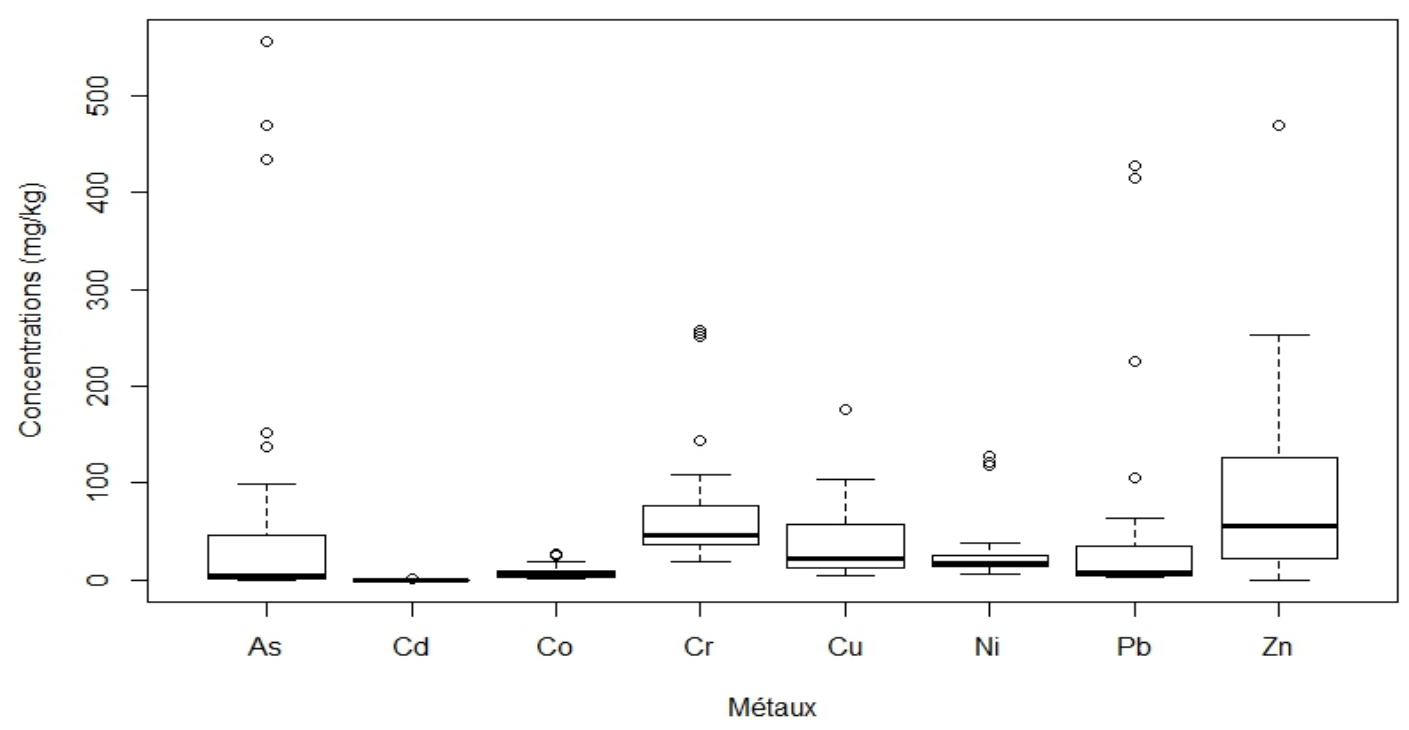

Figure 3: Concentrations en ETM dans les échantillons des sols prélevés dans la zone de Komabangou.

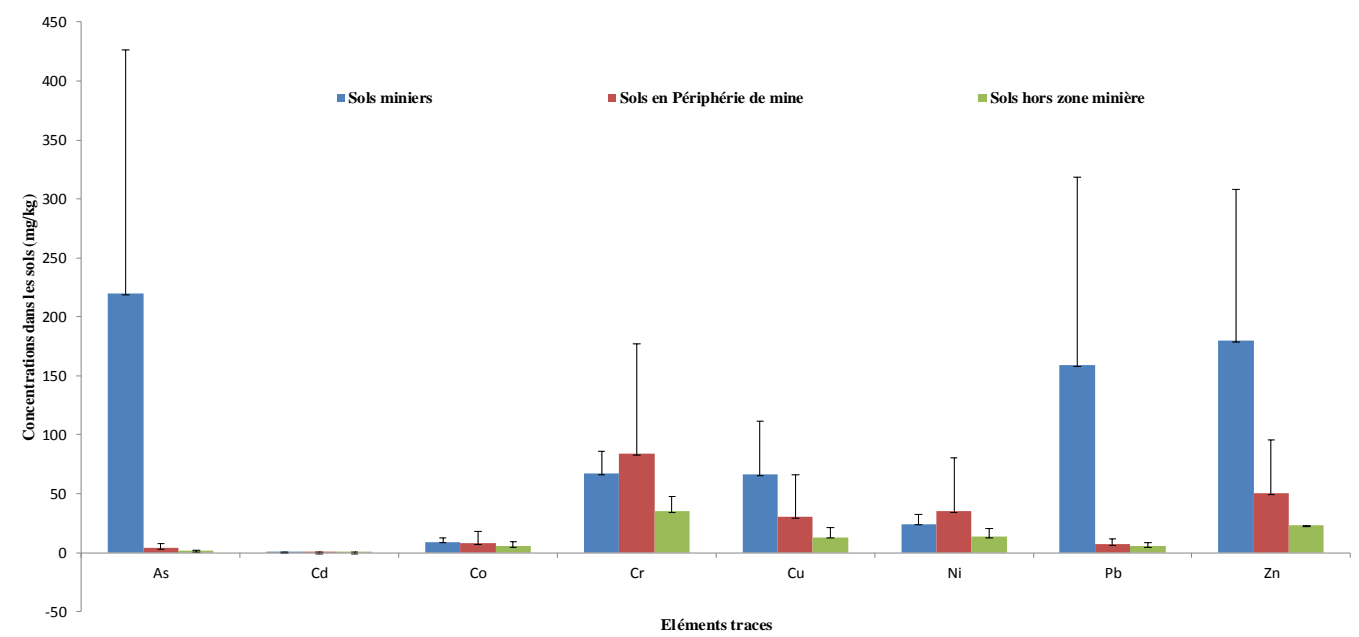

Figure 4 : Concentrations en ETM dans les sols de Komabangou en fonction des sites de prélèvement. 


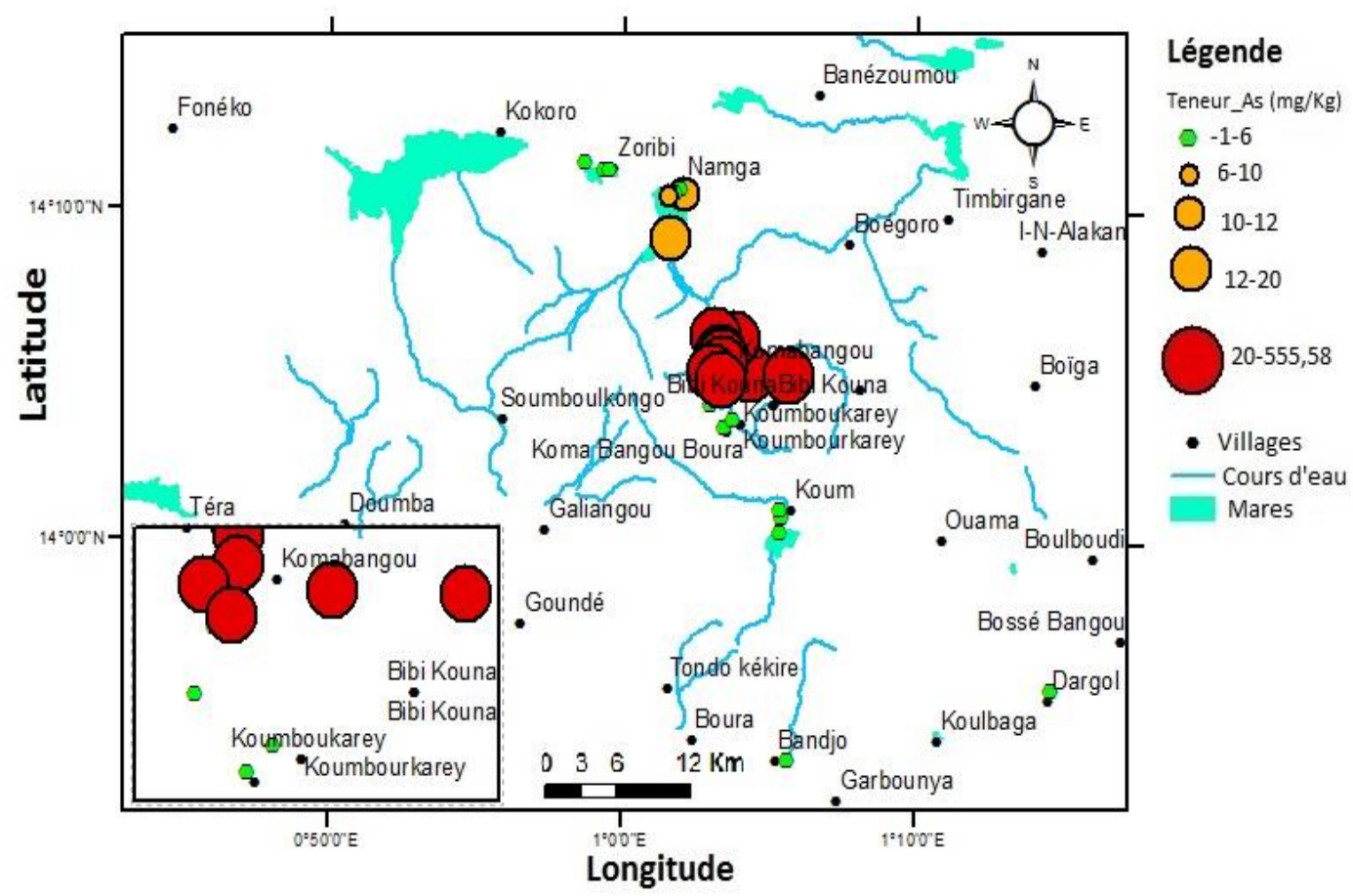

Figure 5 : Répartition spatiale des teneurs en arsenic dans les sols.

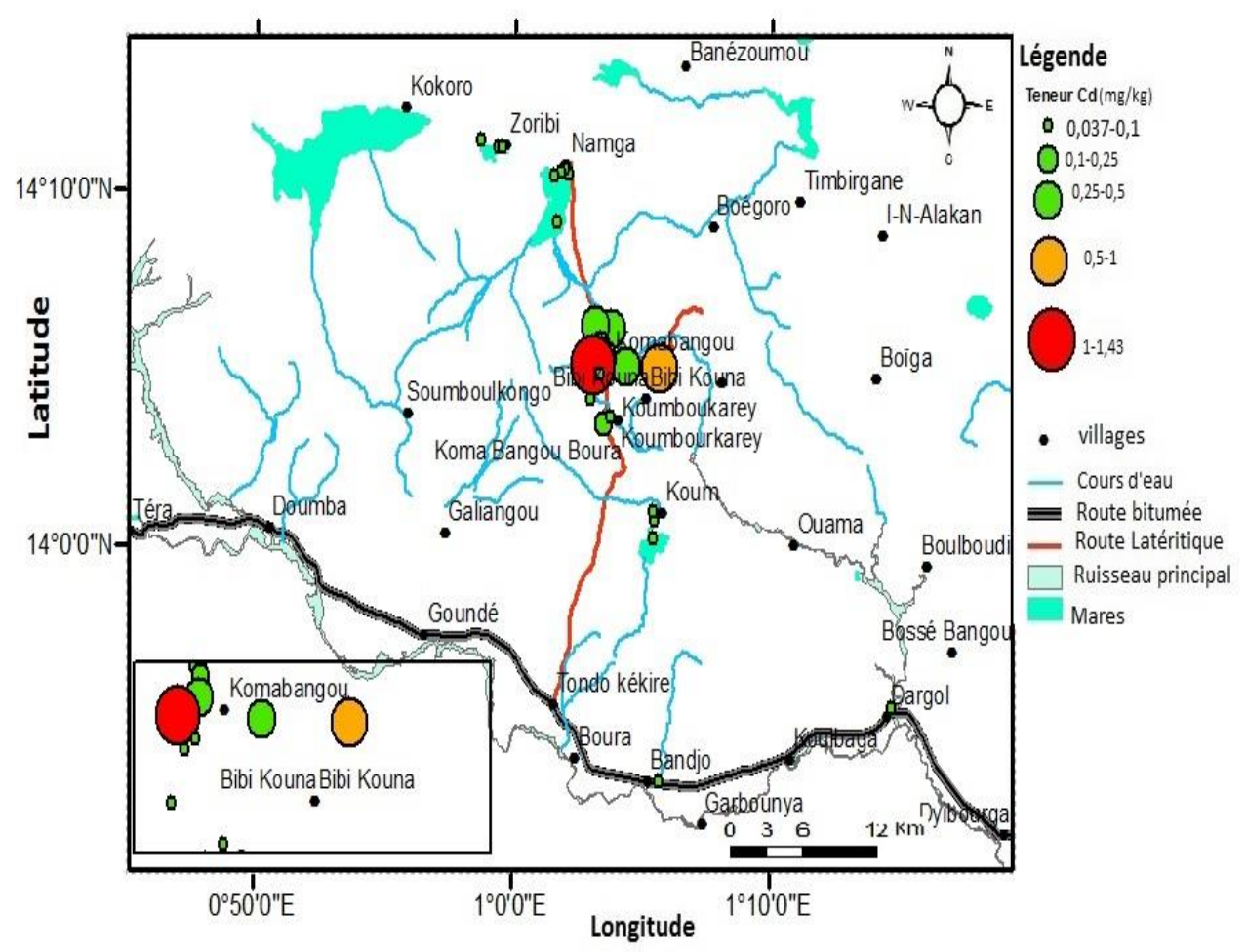

Figure 6: Répartition spatiale des teneurs en cadmium dans les sols. 


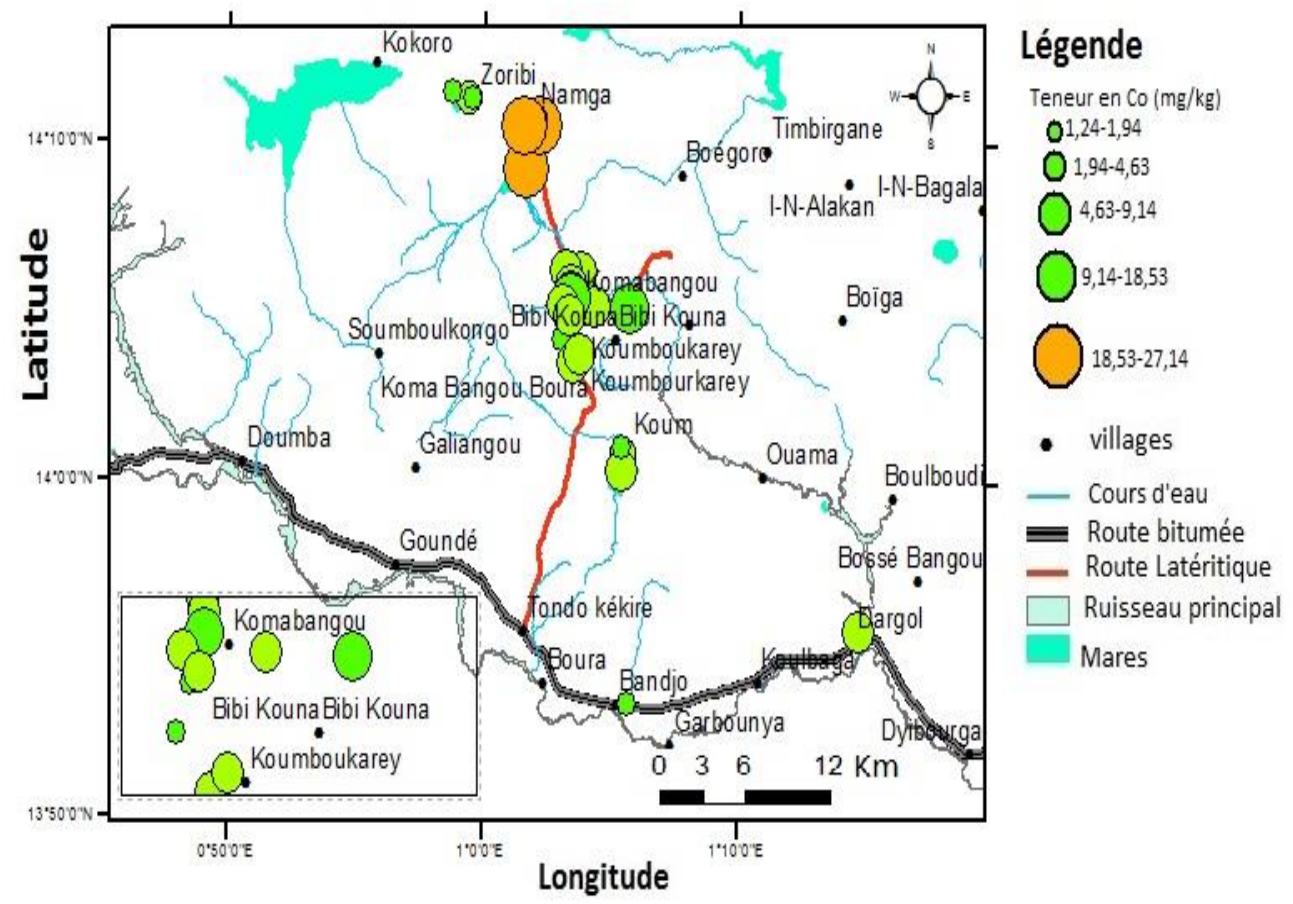

Figure 7 : Répartition spatiale des teneurs en Co dans les sols.

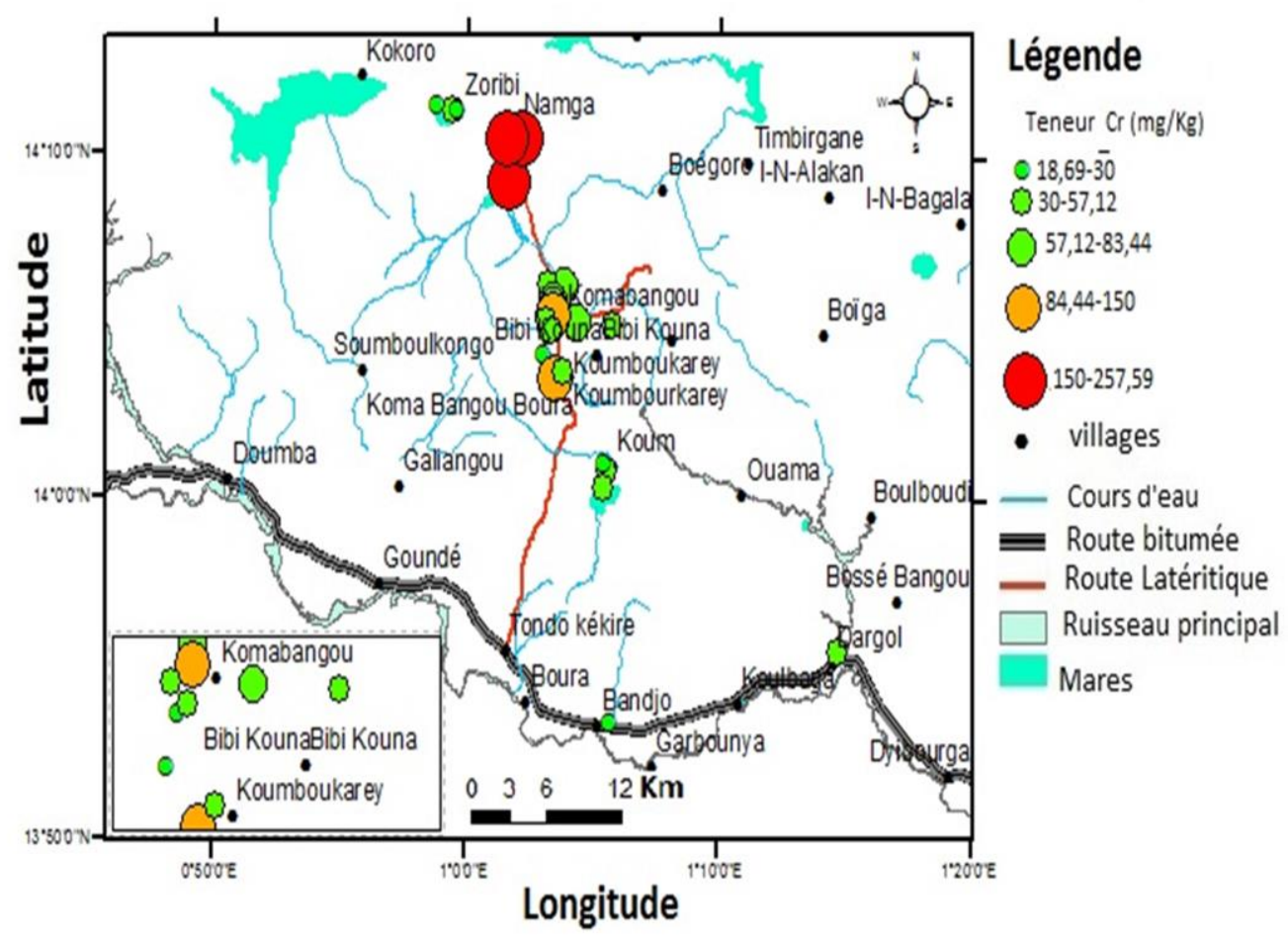

Figure 8 : Répartition spatiale des teneurs en $\mathrm{Cr}$ dans les sols. 


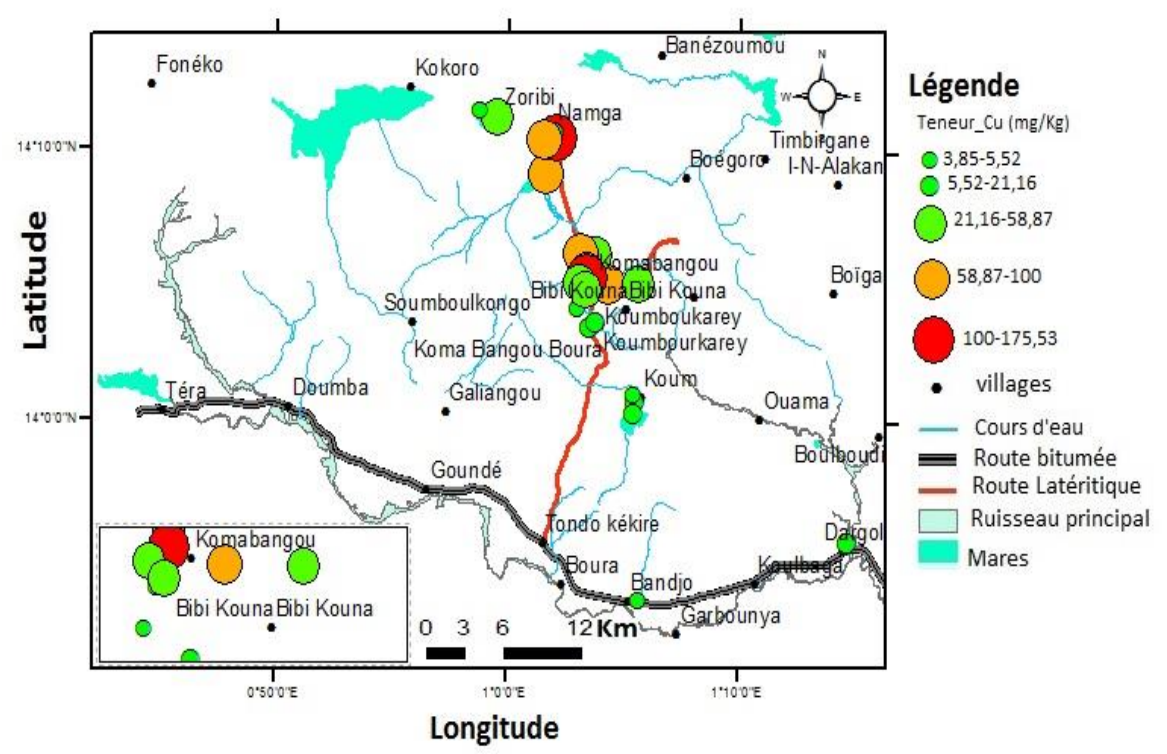

Figure 9 : Répartition spatiale des teneurs en $\mathrm{Cu}$ dans les sols.

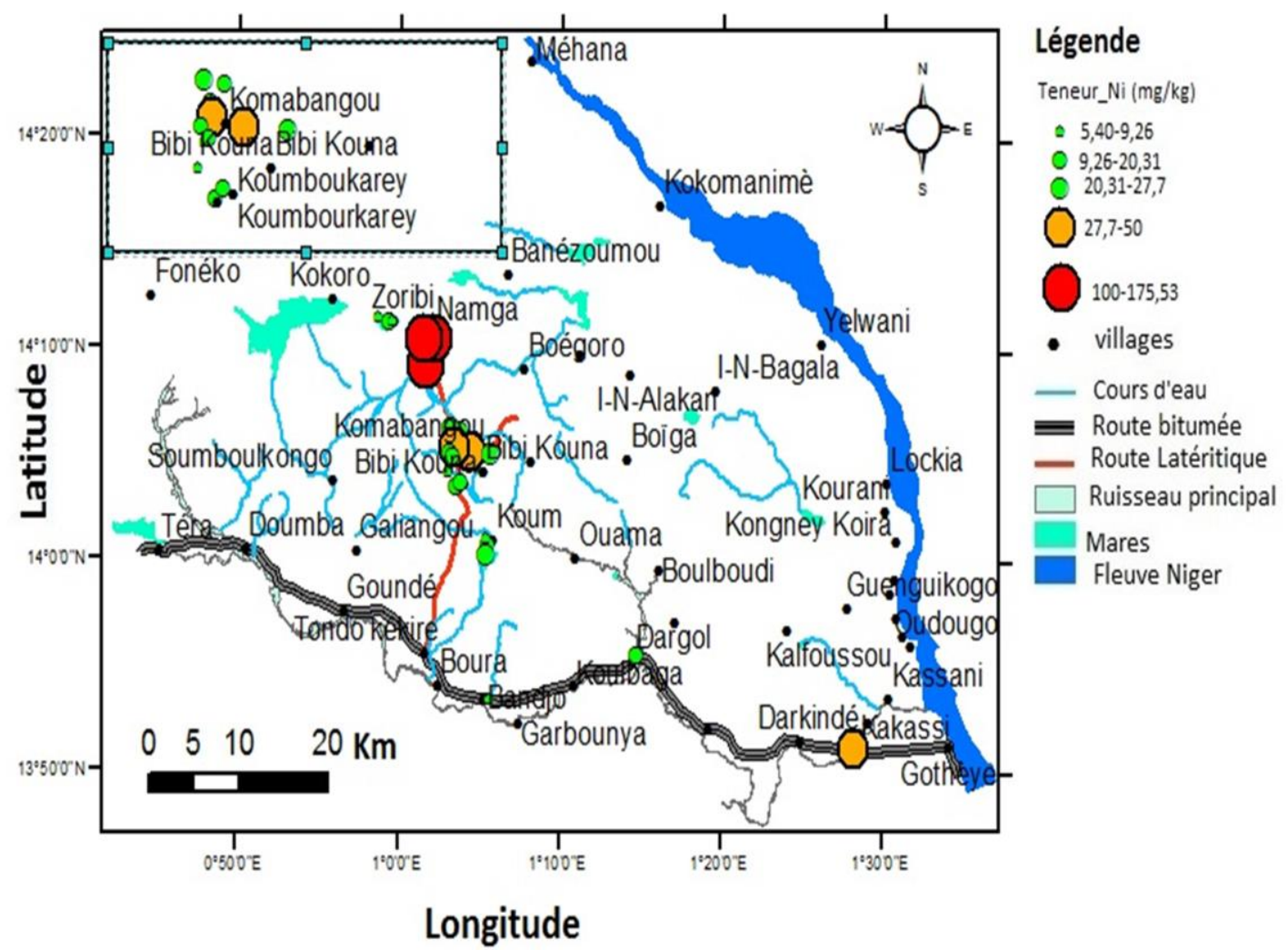

Figure 10 : Répartition spatiale des teneurs en Ni dans les sols. 


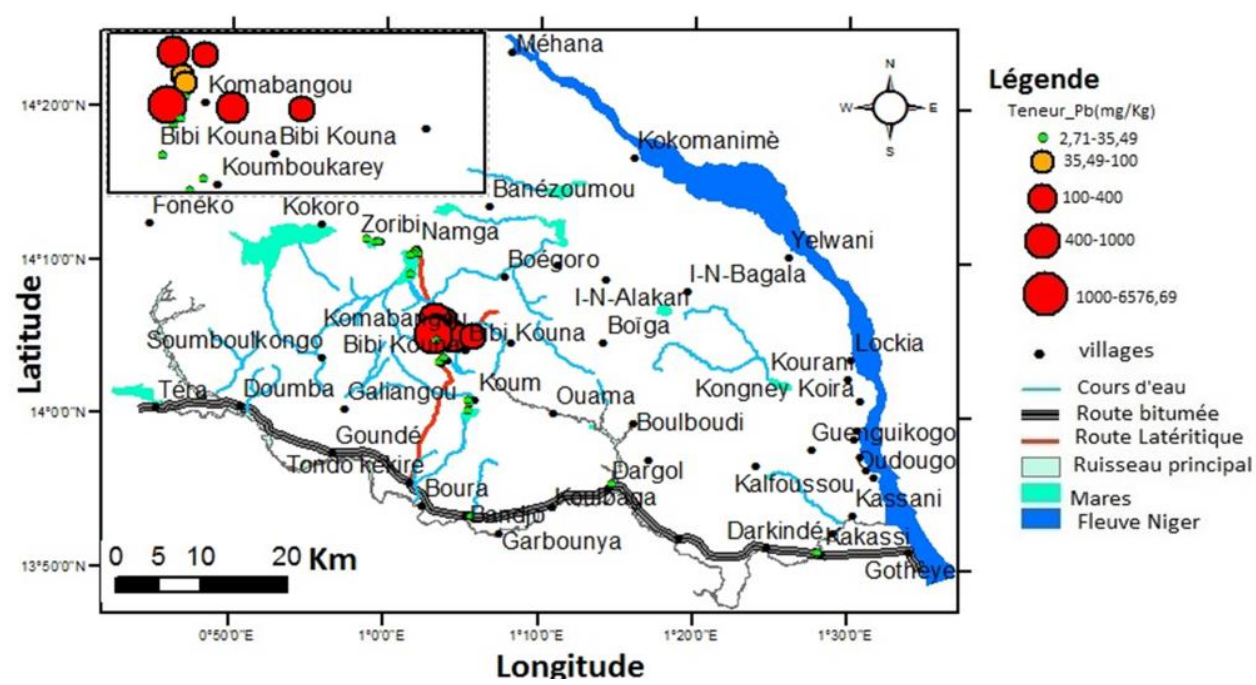

Figure 11 : Répartition spatiale des teneurs en $\mathrm{Pb}$ dans les sols.

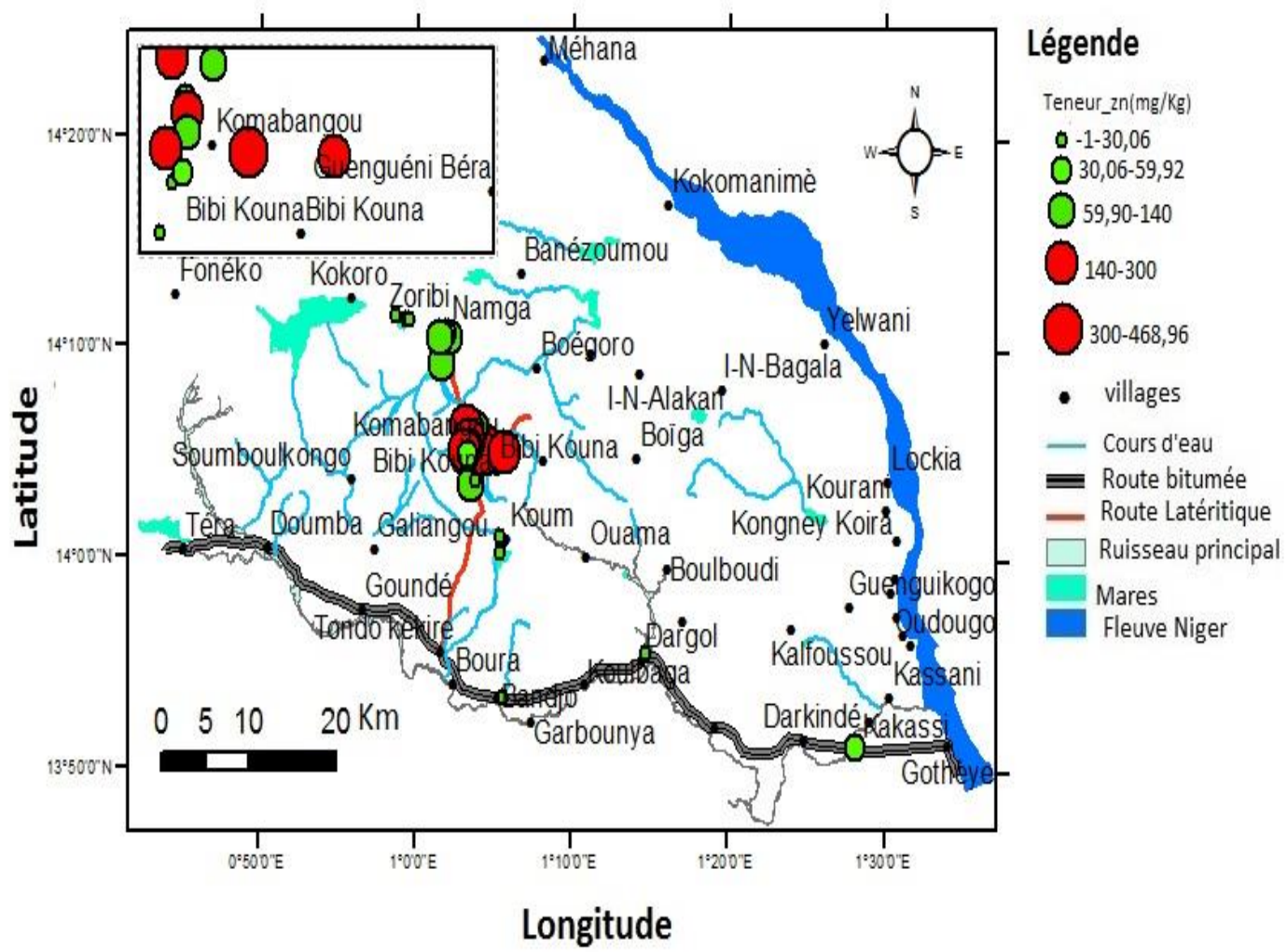

Figure 12 : Répartition spatiale des teneurs en $\mathrm{Zn}$ dans les sols. 


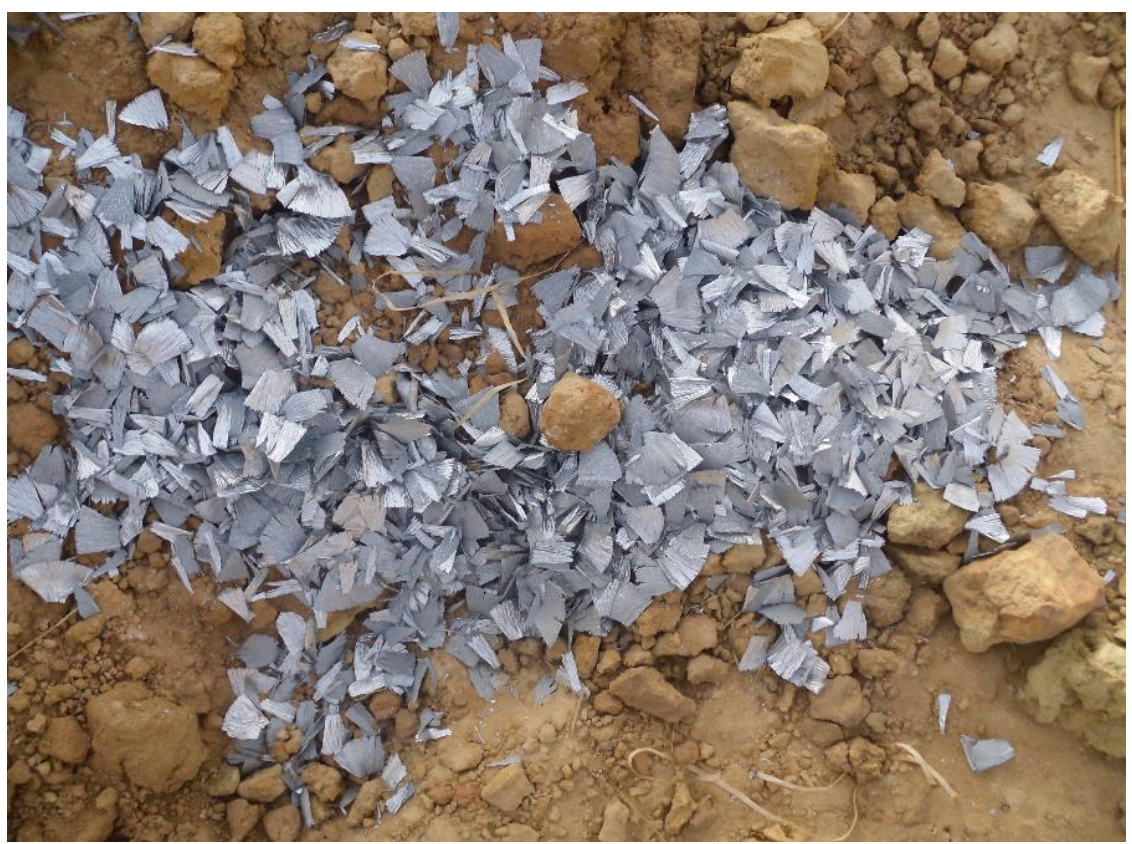

Figure 13 : Copeaux de zinc déversés au sol

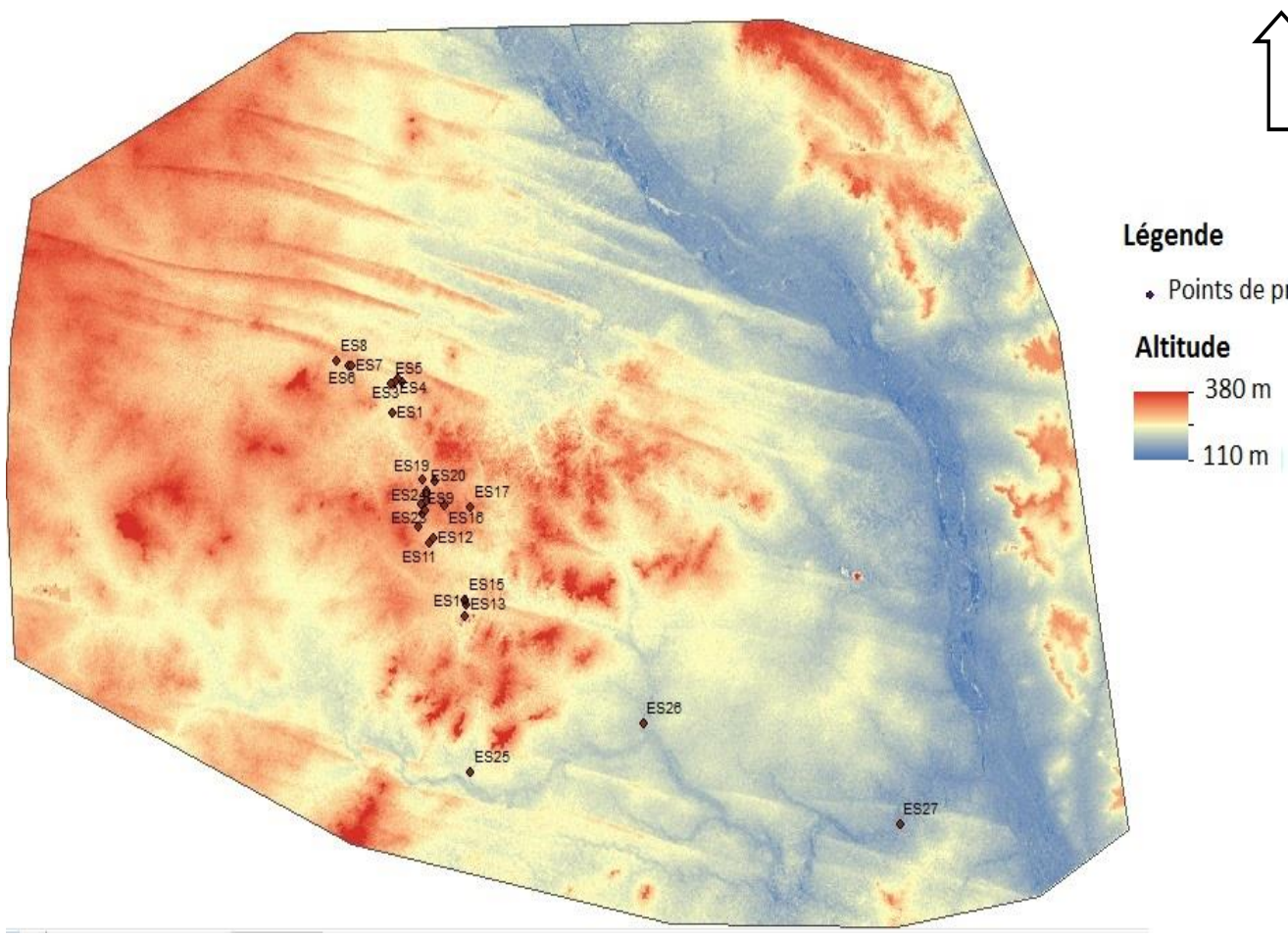

Figure 14: Topographie de la zone de Komabangou. 
Tableau 1 : Index de pollution dans les sols.

\begin{tabular}{|c|c|c|c|}
\hline Sites & Catégorie du sol & Echantillons & Index de pollution \\
\hline Namga & Sol zone périphérique & ES1 & 1,11 \\
\hline Namga & Sol zone périphérique & ES2 & 1,13 \\
\hline Namga & Sol zone périphérique & ES3 & 0,20 \\
\hline Namga & Sol zone périphérique & ES4 & 0,14 \\
\hline Namga & Sol zone périphérique & ES5 & 1,03 \\
\hline Zoribi & Sol zone périphérique & ES6 & 0,16 \\
\hline Zoribi & Sol zone périphérique & ES7 & 0,17 \\
\hline Kokorou & Sol zone périphérique & ES8 & 0,06 \\
\hline Forage Komabangou & Sol zone périphérique & ES9 & 0,15 \\
\hline Forage soumboukoungou & Sol zone périphérique & ES10 & 0,12 \\
\hline Boura & Sol zone périphérique & ES11 & 0,43 \\
\hline Boura & Sol zone périphérique & ES12 & 0,24 \\
\hline Kounhou & Sol zone périphérique & ES13 & 0,26 \\
\hline Kounhou & Sol zone périphérique & ES14 & 0,24 \\
\hline Kounhou & Sol zone périphérique & ES15 & 0,09 \\
\hline Grillage El Saadou & Sol zone minière & ES16 & 4,43 \\
\hline Grillage Bidon & Sol zone minière & ES17 & 12,31 \\
\hline Grillage Morey & Sol zone minière & ES18 & 10,26 \\
\hline Grillage Baba Djallo & Sol zone minière & ES19 & 3,10 \\
\hline Nakia Sossoriya & Sol zone minière & ES20 & 1,10 \\
\hline Maijamaa Sossoriya & Sol zone minière & ES21 & 1,33 \\
\hline Abdallah Sossoriya & Sol zone minière & ES22 & 3,49 \\
\hline Banbara Sosssriya & Sol zone minière & ES23 & 17,84 \\
\hline Gawado Sossoriya & Sol zone minière & ES24 & 1,35 \\
\hline Bandjo & Sol hors zone minière & ES25 & 0,06 \\
\hline Dargol & Sol hors zone minière & ES26 & 0,22 \\
\hline Kakassi & Sol hors zone minière & ES27 & 0,46 \\
\hline
\end{tabular}




\section{DISCUSSION \\ Corrélations entre les différents métaux lourds étudiés}

Il existe des corrélations entre les différents éléments traces métalliques étudiés (Tableau 2). Les fortes corrélations sont enregistrées entre les éléments $\mathrm{Co}, \mathrm{Cr}, \mathrm{Cu}$ et $\mathrm{Ni}$ indiquant de fortes probabilités de les rencontrer ensemble sur les différents sites. Ces corrélations pourraient être dues à une anomalie géochimique d'influence ultramafique de la zone. Une forte concentration en $\mathrm{Ni}$ sur un site, par exemple, signifie une forte concentration en $\mathrm{Cu}$, en $\mathrm{Co}$, en $\mathrm{Cr}$ et vice versa. C'est l'exemple du site de Namga avec les échantillons ES1, ES2 et ES5. Les index de pollution (Tableau 3) calculés à partir de l'équation $\mathrm{IP}=$ $[(\mathrm{Co} / 30+\mathrm{Cr} / 150+\mathrm{Cu} / 100+\mathrm{Ni} / 50) / 4]$ donne des IP $>1$ dans cette zone de Namga révélant ainsi la contamination multiple de ses sols par le $\mathrm{Co}, \mathrm{Cu}, \mathrm{Cr}, \mathrm{Ni}$.

Les sites Grillage bidon, Grillage Morey, Abdallah Sossoriya, avec des IP respectifs de 12,31;10,26; 17,84; (du fait principalement de leurs teneurs anormalement élevées en ETM) sont qualifiés de très fortement pollués. Smouni et al. (2010) rapportent des résultats d'IP variant de 0,08 à 47,69 dans une zone minière du Maroc oriental comprenant des gammes des valeurs d'IP assez proches de celles obtenues à Komabangou.

\section{Comparaison des teneurs en ETM des échantillons de sol aux valeurs règlementaires}

Les concentrations en ETM des différents échantillons de sol prélevés à Komabangou sont données dans le tableau 4. Les huit éléments traces métalliques recherchés à savoir $\mathrm{As}, \mathrm{Cd}, \mathrm{Co}, \mathrm{Cr}, \mathrm{Cu}, \mathrm{Ni}, \mathrm{Pb}$ et $\mathrm{Zn}$ ont été détectés sur tous les sites. Les valeurs de concentrations obtenues varient en fonction du site de prélèvement et de l'élément métallique considéré. En moyenne la séquence des teneurs en ETM dans les échantillons est $\mathrm{Pb}>\mathrm{Zn}>\mathrm{As}>\mathrm{Cr}>\mathrm{Cu}>$ $\mathrm{Ni}>\mathrm{Co}>\mathrm{Cd}$. Ce résultat suggère que la contamination du sol dépend de l'ampleur des activités ainsi que de l'importance de la superficie d'orpaillage mais aussi des produits chimiques utilisés (exemple du site du procédé par cyanuration Grillage El Saadou dont l'échantillon ES16 prélevé a enregistré la plus grande concentration en zinc). On constate, au niveau des échantillons (ES25, ES26, ES27) des sols hors zone minière, qu'aucune concentration supérieure ou sensiblement égale à la valeur normale n'est enregistrée.

Les plus fortes concentrations en ETM obtenues sont détectées dans la zone minière suggérant que l'orpaillage est une source de pollution des sols. En effet, les teneurs élevées en $\mathrm{Pb}$ s'expliqueraient par le fait que les minerais d'or sont souvent associés aux minerais sulfureux parmi lesquels on peut citer la galène $(\mathrm{PbS})$, principal minerai $\mathrm{du} \mathrm{Pb}$. Les teneurs élevées en $\mathrm{Zn}$ peuvent s'expliquer non seulement par le fait que les minerais de $\mathrm{Zn}$ comme la blende ( $\mathrm{ZnS})$ sont intimement associés aux minerais d'or mais aussi par l'utilisation de copeaux de zinc pour la cémentation de l'or; copeaux qui sont parfois déversés au sol sur les sites miniers (Figure 13).

\section{Risques liés à la pollution des sols de Komabangou par les ETM}

Le sol de Komabangou pollué en ETM devient à son tour une source possible de diffusion directe ou indirecte de ces polluants dans l'environnement, via l'eau, les envols de poussières, etc., où ils deviennent dangereux car potentiellement absorbés par les êtres vivants. La topographie de la zone de Komabangou (figure 14) montre que les parties polluées en ETM sont en hautes altitudes. Les eaux de pluie peuvent lessiver les sols pollués et migrer vers les points de basses altitudes où se trouvent le fleuve Niger et plusieurs cours qui risquent ainsi d'être pollués. Les ETM peuvent être également bioaccumulés dans les tissus des plantes et induire des perturbations au niveau de leur métabolisme (Cheng, 2003). À la suite du phénomène de bioconcentration, les ETM peuvent, en effet, se retrouver dans les végétaux à des concentrations supérieures aux concentrations présentes dans le milieu (Taylor et McLennan, 1981). Il est à noter qu'une accumulation d'ETM dans une plante ne se traduira pas nécessairement pas une 
altération de la santé de la plante ou par l'apparition de symptômes visibles de contamination (Taylor et McLennan, 1981). L'effet toxique de ces éléments varie avant tout selon le type de métal présent, sa concentration dans la plante, le temps d'exposition et selon l'espèce végétale affectée. Les ETM peuvent donc en cas de cultures dans la zone de Komabangou s'accumuler dans les plantes et constituer un danger pour leur métabolisme.

\section{Conclusion}

Dans cette étude, la distribution des éléments traces dans les sols de la zone aurifère de Komabangou a été investiguée. Les cartes de répartition spatiale montrent clairement une importante concentration des éléments tels que $\mathrm{As}, \mathrm{Cd}, \mathrm{Pb}$ et $\mathrm{Zn}$ au centre de la zone aurifère. Dans la majorité des cas, les concentrations en ETM décroissent dans les sols dans l'ordre suivant: site minier, site en périphérie de mine et site hors zone minière suggérant ainsi l'impact de l'exploitation aurifère sur la pollution des sols par ces ETM. Plusieurs de ces teneurs en ETM sont supérieures aux seuils réglementaires. 12 sites sur 27 étudiés, ont des valeurs d'IP supérieures à 1 , révélant ainsi une contamination des sols par plusieurs éléments. Cette accumulation des métaux dans les sols pourrait poser de sérieux risques sanitaires pour la population vivant dans et aux alentours de la zone aurifère de Komabangou d'où la nécessité de la mise en place d'un plan de surveillance de la contamination des sols et d'envisager la dépollution de ces sols.

\section{CONFLIT D'INTERETS}

Les auteurs déclarent qu'ils n'ont pas de conflit d'intérêts.

\section{CONTRIBUTIONS DES AUTEURS}

$\mathrm{CF}$, TS et GE ont participé à l'élaboration du protocole. OZI, ATD, GY ont participé à l'élaboration du protocole, supervisé sa mise application et la rédaction de l'article. FMMI a participé à la collecte des données.

\section{REFERENCES}

Adeyi AA, Babalola BA. 2017. Lead and cadmium levels in residential soils of
Lagos and Ibadan, Nigeria. Journal of Health and Pollution, 7(13): 42-55. DOI:10.5696/2156-9614-7-13.42.

Agusa T, Kunito T, Fujihara J, Kubota R, Minh TB, Trang PTK, Tanabe S. 2006. Contamination by arsenic and other trace elements in tube-well water and its risk assessment to humans in Hanoi, Vietnam. Environmental Pollution, 139(1) : $\quad 95-106 . \quad$ DOI: 10.1016/j.envpol.2005.04.033

Aucejo A, Ferrer J, Gabaldón C, Marzal P, Seco A. 1997. Diagnosis of boron, fluorine, lead, nickel and zinc toxicity in citrus plantations in Villarreal, Spain. Water, Air, and Soil Pollution, 94(3-4) : 349-360. DOI: 10.1007/BF02406067.

Baize D, Paquereau H. 1997. Teneurs totales en éléments traces dans les sols agricoles de Seine-et-Marne. Etude et Gestion des Sols, 4: 77-94.

Bougherara H, Zdero R, Dubov A, Shah S, Khurshid S, Schemitsch EH. 2011. A preliminary biomechanical study of a novel carbon-fibre hip implant versus standard metallic hip implants. Medical Engineering and Physics, 33(1): 121128.

DOI:

10.1016/j.medengphy.2010.09.011

Breuer M, Ditrich K, Habicher T, Hauer B, Kesseler M, Stürmer R, Zelinski $T$. 2004. Industrial methods for the production of optically active intermediates. Angewandte Chemie International Edition, 43(7): 788-824. DOI: 10.1002/anie.200300599

Cheng S. 2003. Heavy metal pollution in China: origin, pattern and control. Environmental Science and Pollution Research, 10(3): 192-198. DOI: 10.1065/espr2002.11.141.1

Chon HT, Ahn JS, Jung MC. 1998. Seasonal variations and chemical forms of heavy metals in soils and dusts from the satellite cities of Seoul, Korea. Environmental Geochemistry and Health, 20(2): 77-86. DOI: 10.1023/A:1006593708464

Chouti WK, Adanve M, Mama D. 2018. Dosage du plomb et du zinc dans les cultures de l'amarante (Amarantus cruentus) et de la Grande morelle (Solanum macrocarpum): cas de 
quelques sites maraîchers de PortoNovo. Int. J. Biol. Chem. Sci., 12(5): 2381-2395. DOI: 10.4314/ijbcs.v12i5.36

INS. 2010. Institut National de la Statistique, Rapport de l'estimation de la population du Niger, 30p.

Jaishankar M, Tseten $\mathrm{T}$, Anbalagan $\mathrm{N}$, Mathew BB, Beeregowda KN. 2014. Toxicity, mechanism and health effects of some heavy metals. Interdisciplinary Toxicology, 7(2): 60-72. DOI: 10.2478/intox-2014-0009.

Lamine C. 2011. Anticiper ou temporiser: injonctions environnementales et recompositions des identités professionnelles en céréaliculture. Sociologie du travail, 53(1): 75-92.

Lanphear BP, Hornung R, Khoury J, Yolton $\mathrm{K}$, Baghurst P, Bellinger DC, Greene T. 2005. Low-level environmental lead exposure and children's intellectual function: an international pooled analysis. Environmental Health Perspectives, 113(7): 894. DOI: 10.1289/ehp.7688

Lee CS, Li X, Shi W, Cheung SC, Thornton I. 2006. Metal contamination in urban, suburban, and country park soils of Hong Kong: A study based on GIS and multivariate statistics. Science of the Total Environment, 356(1): 45-61. DOI: 10.1016/j.scitotenv.2005.03.024

Lu Y, Song S, Wang R, Liu Z, Meng J, Sweetman AJ, Luo W. 2015. Impacts of soil and water pollution on food safety and health risks in China. Environment International, 77: 5-15. DOI: 10.1016/j.envint.2014.12.010

Oymak T, Ulusoy H, Hastaoğlu E, Yılmaz V, Yıldırım Ş. 2017. Some Heavy Metal

Contents of Various Slaughtered Cattle Tissues in Sivas-Turkey. JOTCSA, 4(3): 737-46. DOI: 10.18596/jotcsa.292601

Rahman R, Asombang AW, Ibdah JA. 2014. Characteristics of gastric cancer in Asia. World Journal of Gastroenterology, 20(16):44-83.

DOI: 10.3748/wjg.v20.i16.4483.
Smouni A, Ater M, Auguy F, Laplaze L, El Mzibri M, Berhada F, Doumas P. 2010. Évaluation de la contamination par les éléments-traces métalliques dans une zone minière du Maroc oriental. Cahiers Agricultures, 19(4): 273-279. DOI: 10.1684/agr.2010.0413

Tankari Dan-Badjo A, Guero Y, Dan Lamso N, Tidjani AD, Ambouta KJM, Feidt C, Sterckeman T, Echevarria G. 2013. Evaluation de la contamination des sols par les éléments traces métalliques dans les zones urbaines et périurbaines de la ville de Niamey (Niger). Revue des BioRessources, 2(2) : 82-95.

Tankari Dan-Badjo A, Tidjani DA, Idder T, Guero Y, Dan Lamso N, Matsallabi A, Echevarria G. 2014. Diagnostic de la contamination des eaux par les éléments traces métalliques dans la zone aurifère de Komabangou-Tillabéri, Niger. Int. J. Biol. Chem. Sci., 6(8): 28-49. DOI: 10.4314/ijbcs.v8i6.41

Taylor SR, McLennan S. 1981. The composition and evolution of the continental crust: rare earth element evidence from sedimentary rocks. Phil. Trans. R. Soc. Lond. A, 301(1461) : 381399. DOI: $10.1098 /$ rsta.1981.0119

Toure M, Tiho S, Ehouman NM, Kpan Kpan KG. 2017. Distribution et structure des communautés de vers de terre et leur corrélation avec des éléments traces métalliques (ETM) le long des bordures de l'autoroute du Nord en Côte-d'Ivoire. Int. J. Biol. Chem. Sci., 11(5): 2277-2293. DOI: 10.4314/ijbcs.v11i5.26

Toure A, Garat A, Diop C, Cabral M, Epote MJ, Leroy E, Fall M, Diouf A, Dehon B, Allorge D. 2016. Présence de métaux lourds et de résidus médicamenteux dans les effluents des établissements de santé de Dakar (Sénégal). Int. J. Biol. Chem. Sci., 10(3): 1422-1432. DOI: 10.4314/ijbcs.v10i3.40. 